\title{
The Use of Autologous Fat Grafting and Platelet Rich Plasma in the Treatment of Chronic Wounds
}

\author{
Robert Bowen MD*, Ginna Treadwell RN MBA, Jason Turner MD and Liveo Romani MD \\ WVU School of Medicine, Center for Wound Care and Hyperbaric Medicine, Berkeley Medical Center, USA
}

*Corresponding author: Robert Bowen, WVU School of Medicine, Center for Wound Care and Hyperbaric Medicine, Berkeley

Medical Center, Martinsburg, USA

\begin{abstract}
Chronic wounds resulting in tissue loss/necrosis present a difficult clinical challenge. Traditional techniques such as wound packing and vacuum therapy are not always sufficient to result in complete closure of the wound. Cell and growth factor assisted fat grafting has been successfully used for restoring loss of volume in cosmetic surgery patients. Cases are presented where chronic wounds with tissue defects were treated using cell and growth factor (PRP) assisted fat grafting. Three patients with chronic wounds resulting in tissue defects which were refractory to debridement, packing, and wound vacuum therapy underwent adipose tissue harvesting with liposuction, isolation of stromal vascular fraction (SVF) from the adipose tissue and peripheral blood drawn to obtain platelet rich plasma (PRP). Adipose tissue enriched with SVF and PRP was introduced into the wound and peri-wound and the patients were followed and their course documented with serial photographs and ultrasound examinations. In all cases, previously refractory wounds healed with deep tissue regeneration and re-epithelialization as determined by ultrasound examination and digital photography. Duration of wounds prior to grafting: mean 163days +/- 49. Time to complete closure following grafting: 42.2 days +/- 7.2Cell and growth factor enriched adipose tissue is shown to be a viable "treatment of the last resort" for chronic refractory wounds resulting in tissue loss. Further investigation in clinical trials may be warranted.
\end{abstract}

\section{Background}

Chronic wounds resulting in tissue loss/necrosis present a difficult clinical challenge. Traditional approaches including gauze packing or vacuum therapy are often not sufficient to close such wounds. Autologous fat grafting is a technique which has been employed in reconstructive and cosmetic surgery for restoring volume loss [1-3] and evidence suggests that the addition of stromal vascular fraction (SVF) and or platelet rich plasma (PRP) to the adipocytes enhances engraftment of the transplanted fat [4- 7]. Three cases are presented in which usual and customary care was insufficient heal wounds with a tissue defect and which were subsequently treated with autologous adipose tissue grafting resulting in complete closure of the wound.

\section{Method}

Three patients ( 2 males, ages 29 and 42 and 1 female age 88) who presented to the WVU Center for Wound Care and Hyperbaric Medicine at Berkeley Medical Center with wounds that were refractory to usual and customary care after at least 4 months of treatment underwent free tissue grafting with autologous adipocytes harvested with nutational infrasonic liposuction (NIL) ( 2 cases) or syringe aspiration ( 1 case). The adipocytes were prepared for grafting under a laminar flow hood by low speed centrifugation $(50 \mathrm{~g})$ and selecting the fat from the lowest (closest to the infranatant) portion in the $50 \mathrm{ml}$ conical tube. This is the densest portion of adipocytes and are thought to be the youngest and most robust. PRP was prepared using a commercial centrifuge and kits (Angel, Arthrex Tampa, FL) from $60 \mathrm{ml}$ of whole blood to produce $2-4 \mathrm{ml}$ of PRP. Patient \#1 also had SVF prepared from lipoaspirate infranatant and the pellet was added to the grafted material [8]. Patient \#2 had a second aliquot of adipocytes pressed through a series of mesh screens (Tulip) to produce clusters of adipocytes in the range of 400 microns size (nanofat). The grafts were then transplanted into the tissue defects and into the peri wound space using sterile technique and covered with a sterile 
nonadherent dressing. Patients \#1 and \#2 who had deeper and more profound tissue loss were imaged using $10 \mathrm{Mhz}$ ultrasound before and 3 weeks post treatment.

\section{Results}

Patient \#1- Nonhealing traumatic wound to right anterior leg. Patient is a 29 year old man whose injury occurred during a CrossFit workout when he "missed" a box jump and impacted his shin on a sharp corner of the wooden box (Figure 1). The injury was exacerbated when the patient was kayaking the next day and was flipped over into the river exposing his open wound to further contamination. He was treated acutely in the emergency room with antibiotics and referred to the wound service at another institution where he was treated with weekly debridement and dressings. He presented to the wound clinic at BMC 8 weeks later when he felt the wound was not healing. The wound was extensively debrided and found to have deep tunneling lateral to the tibeal plateau approximately $5 \mathrm{~cm}$ below the inferior border of the patella. This area was further characterized by ultrasound imaging (Figure 2) The patient was treated with weekly debridement, packing with honey infused gauze and wound vacuum therapy. Pathological examination of the wound debris revealed organized thrombus and liquefied necrotic material. Despite this treatment regimen the wound failed to improve. The patient was offered the alternative of adipose cell grafting or operative debridement as treatment options. He chose free tissue grafting with autologous adipocytes. The flanks were chosen as the donor site, The area was anesthetized using $0.1 \%$ lidocaine, 1:1,000,000 epinephrine and .0083\% NaHCO3 solution. Lipoaspirate was obtained using NIL (Euromi, Verviers, Belgium), 3.5mm "Super G" cannula (MMT, Irvine CA), -14in. Hg suction (1/2atm) and collected into a sterile canister. The infranatant fluid was centrifuged at $400 \mathrm{~g}$ for 4 minutes and a pellet of cells was collected with a pipette. The supernatant (adipose tissue) was centrifuged at $50 \mathrm{~g}$ for 4 minutes. $60 \mathrm{ml}$ of blood was drawn and then centrifuged using the Angel system (Arthrex. Tampa, FL) to obtain $2 \mathrm{ml}$ of PRP. The tibial wound was debrided and $8 \mathrm{ml}$ of concentrated adipocytes, $2 \mathrm{ml}$ of PRP, and $.1 \mathrm{ml} \mathrm{SVF}$ pellet was placed into the tissue defect using a 14ga. blunt cannula and dressed with a sterile nonadherent dressing.

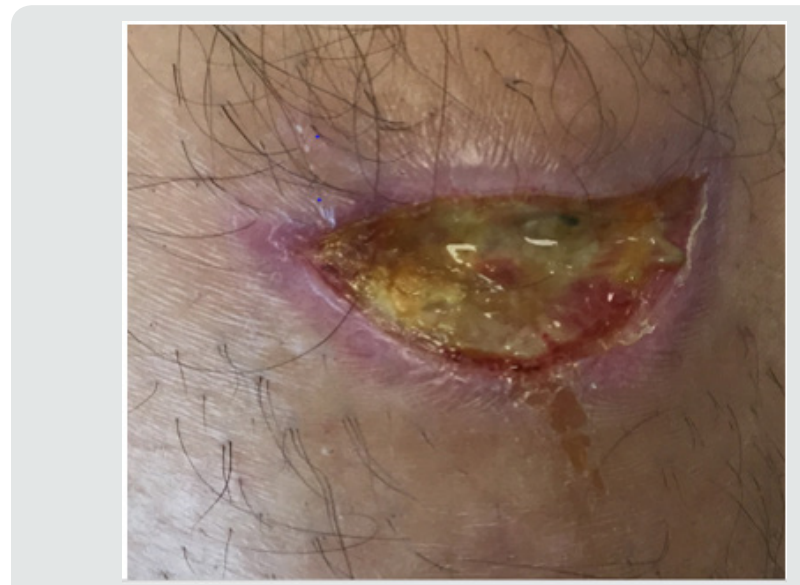

Figure 1: Patient \#1 on presentation.

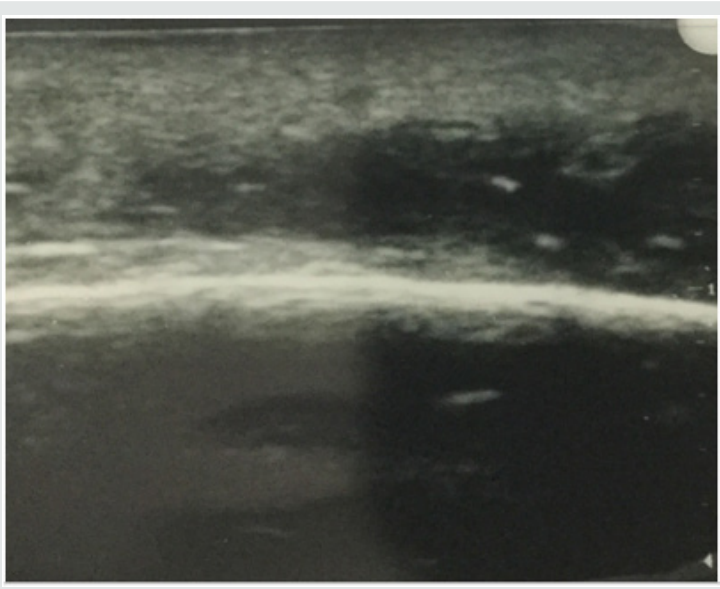

Figure 2: Ultrasound patient \#1 at presentation.

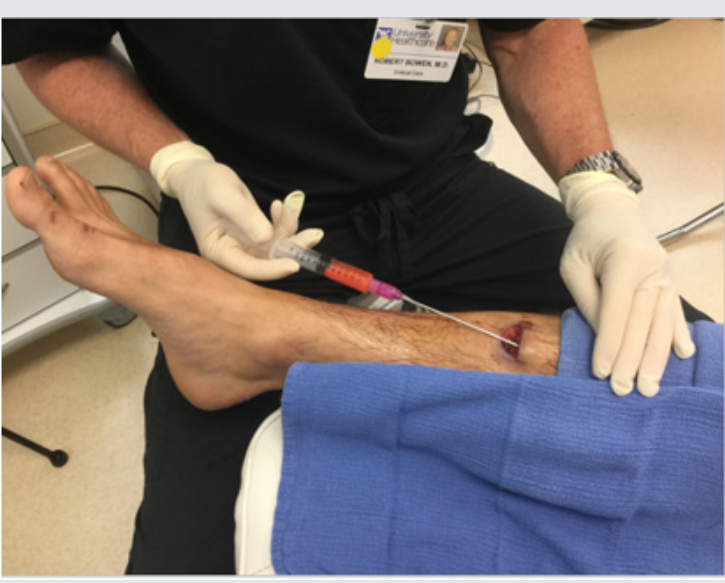

Figure 3: Procedure patient \#1.

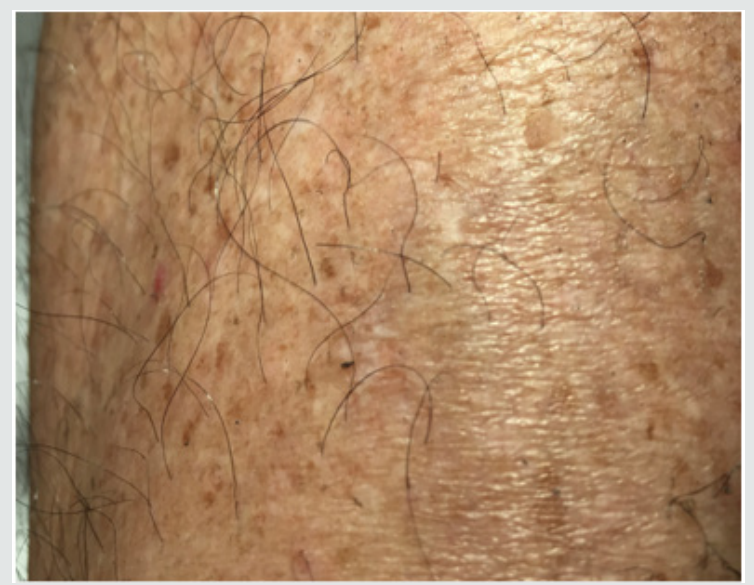

Figure 4: Healed wound patient \#1.

Subsequent weekly visits demonstrated improvement and at 3 weeks following the procedure the patient had complete closure of the wound both cutaneous and subcutaneous (Figure 3). Patient \#2-Pressure ulcer over the greater trochanter. Patient is an 88year old female with Parkinson's disease who developed a pressure ulcer over her left greater trochanter after a 2 week stay in ICU for pneumonia and respiratory failure 6 weeks prior to her presentation at the wound clinic. She was treated with usual and customary care including wound packing and vacuum theory 
but failed to improve over 4 weeks and ultrasound examination demonstrated echolucency consistent with necrosis nearly to the level of cortical bone. The patient consented to autologous free fat grafting and $50 \mathrm{ml}$ of adipose tissue was harvested using a $60 \mathrm{ml}$ Toomey syringe and centrifuged at $50 \mathrm{~g}$ for 4 minutes. $5 \mathrm{ml}$ of the densest and smallest adipocyte clusters were combined with $1 \mathrm{ml}$ of PRP and transplanted into the defect with ultrasound guidance. The patient had healing of the ulcer at 4 weeks evidenced by both skin healing and resolution of tissue defect on ultrasound exam. Patient \#3. Venous ulcer of left lower leg/ankle. 49year old male with refractory venous ulcer that over 16 months had been treated with debridement, compression wrapping, endovascular laser ablation (ELVT), sclerosis of feeder veins and tributaries, and commercial "skin substitutes" (Figures 4-6). (EpiFix, MiMedx, Marietta, GA). He had steady, albeit slow improvement over 16 months but then the healing stalled. The patient consented and underwent autologous free fat grafting. $10 \mathrm{ml}$ of adipose tissue was harvested using tumescent anesthesia (previously described) from the abdominal area using a Toomey syringe and manual suction. $2 \mathrm{ml}$ of PRP was obtained using the previously described protocol. .25ml of PRP was added to $2 \mathrm{ml}$ of the densest adipocytes and grafted into the wound bed and injected into the peri wound area. Over the next 6 weeks compression wraps were continued, and the defect filled in and was reepithelialized. The patient was advanced to compression stockings and at 6 months post procedure remained healed (Figures $7 \& 8)$.

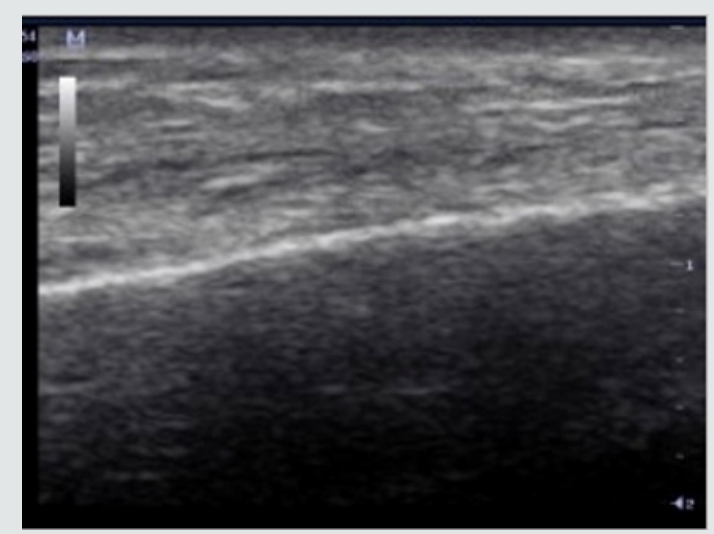

Figure 5: Ultrasound image post treatment patient \#1.

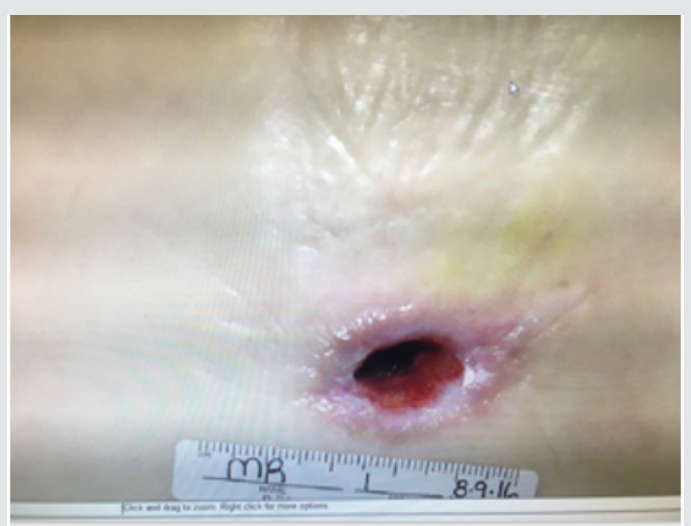

Figure 6: Patient \#2 presentation.

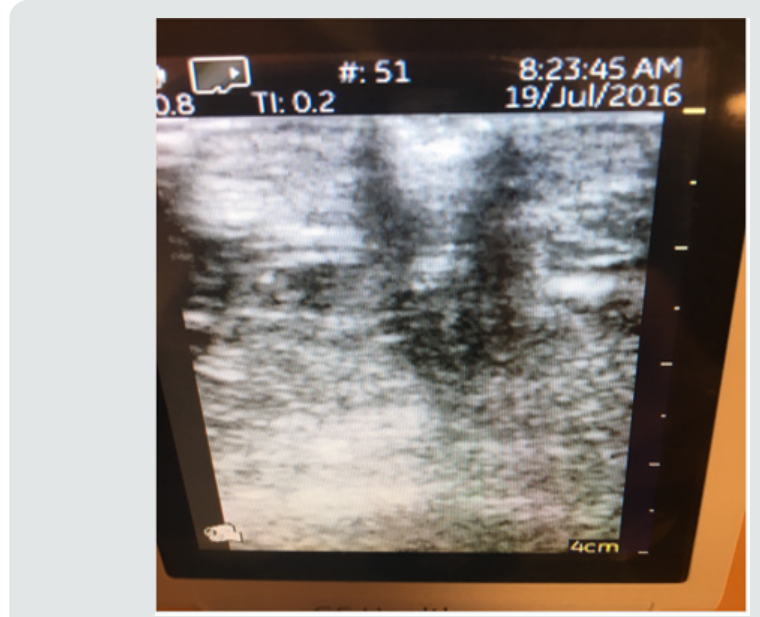

Figure 7: Ultrasound image at presentation patient\#2.

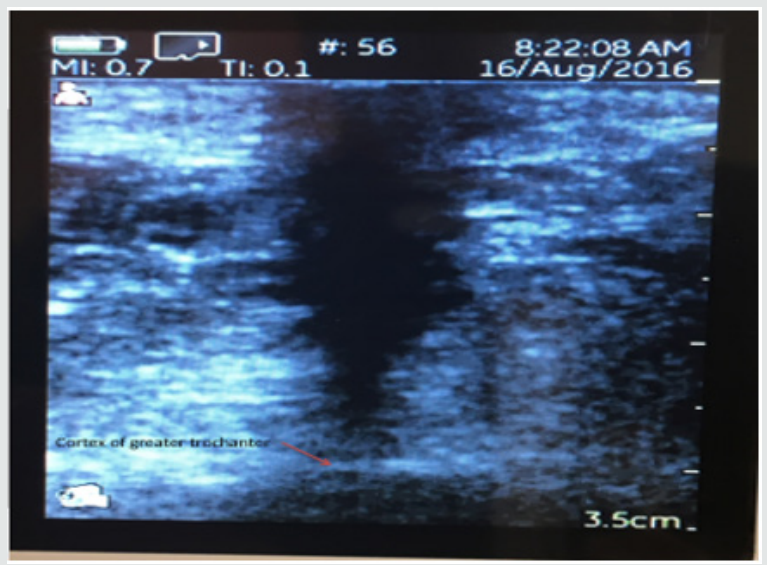

Figure 8: Ultrasound image after standard treatment before grafting patient \#2.

\section{Discussion}

Despite substantial advances in chronic wound care such as negative pressure therapy, advanced wound dressing, hyperbaric oxygen, commercially available skin substitutes, and the proliferation of dedicated wound clinics many chronic wounds heal very slowly or fail to heal at all. Factors accounting for this include significant co-morbidities of cardiovascular disease and diabetes as well as poor compliance (smoking, poor diet, and failure to adhere to offloading). Some wounds fail to heal despite optimization of treatment by health care providers and their patients (Figure 9 \& 10). Autologous fat grafting has been used in cosmetic and reconstructive surgery for restoring volume loss leading to the idea that such an approach could be useful in treating chronic wounds especially those with loss of healthy tissue (Figure $11 \& 12$ ). Adipose tissue has been shown to have not only a volumetric but also a regenerative effect. Adipose derived stem cells (ADSCs) have been shown to produce a regenerative effect through paracrine and autocrine mechanisms as well differentiation into cell types consistent with cells of the host transplant site [9]. Improvement in fat grafting techniques including reduced trauma to grafted tissue and the addition of PRP (source of growth factors) and 
stromal vascular fraction (SVF) derived from adipose tissue by mechanical or enzymatic means have resulted in more predictable and increased uptake of grafted tissue [6-15]. In the first of the three cases reported adipose tissue was enriched with SVF and PRP. However, the isolation of SVF is a time and labor-intensive process and requires infrastructure not commonly employed in operating theaters or procedure rooms. The second and third cases utilized only manually harvested autologous fat and PRP (which can be processed with only a centrifuge) and also resulted in complete healing. In all three cases autologous fat grafting resulted in closure of previously "stalled" wounds refractory to usual and customary care (Figure 13 \& 14).

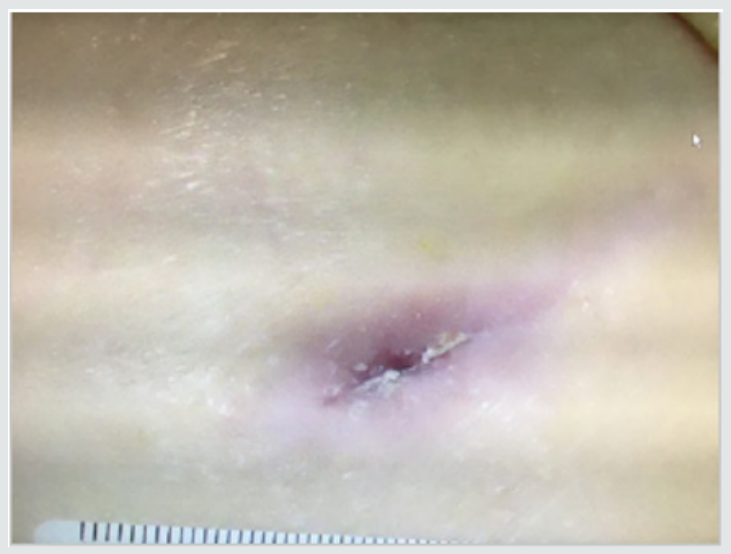

Figure 9: Healed ulcer patient \#2.

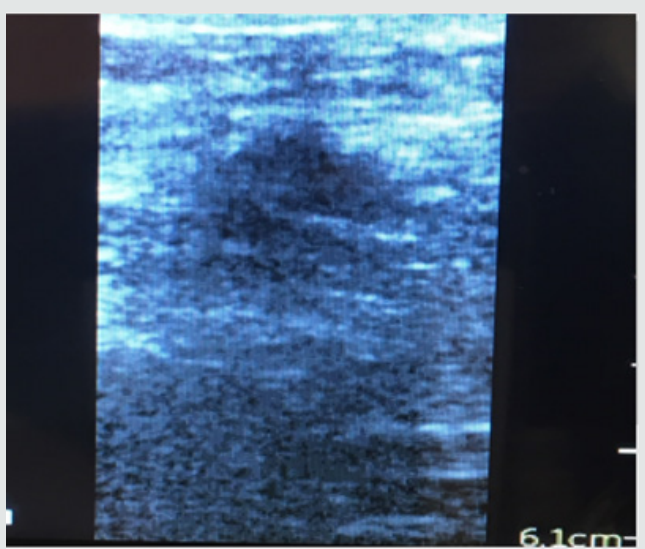

Figure 10: Ultrasound image post grafting patient \#2.

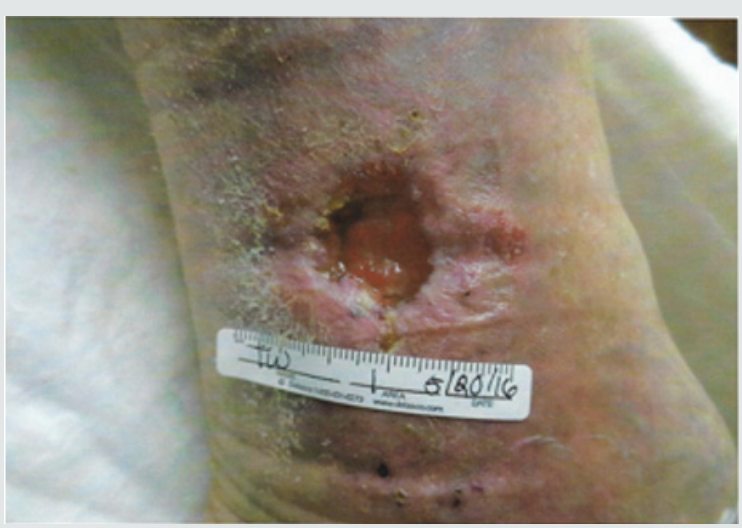

Figure 11: Venous ulcer patient \#3.

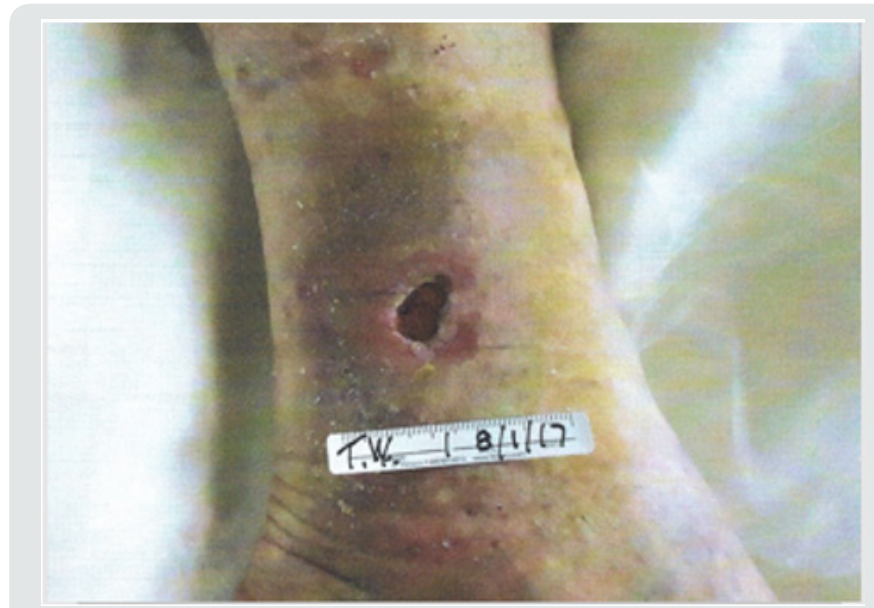

Figure 12: Patient \#3 after vein ablation.

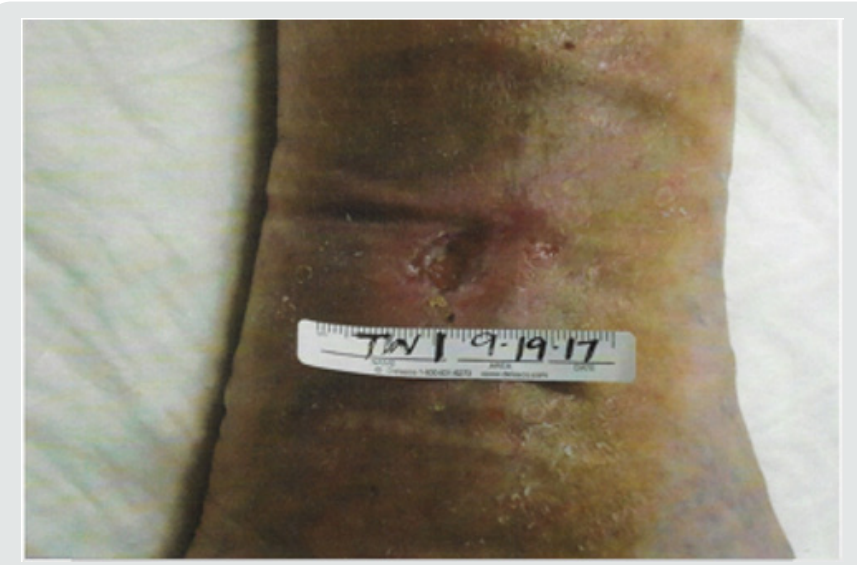

Figure 13: Stalled wound patient \#3.

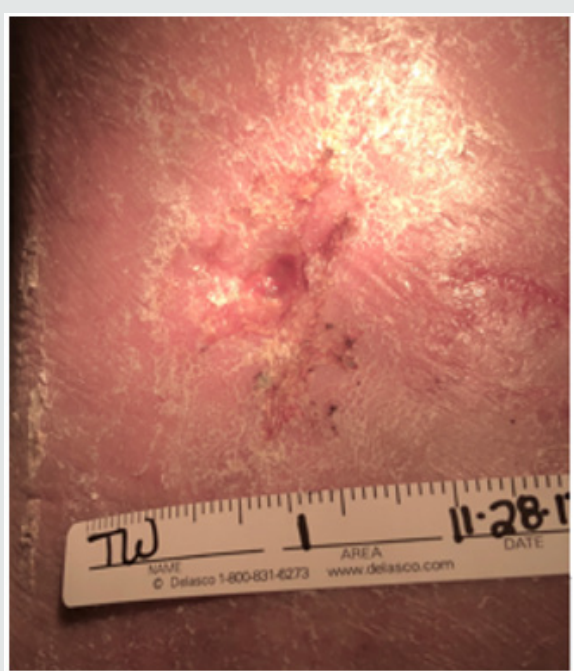

Figure 14: Healed wound post grafting patient \#3.

\section{Conclusion}

Point of care autologous fat grafting combined with PRP can be used successfully as a treatment for chronic wounds refractory to traditional treatment as a treatment of "last resort" without the addition of culture expanded mesenchymal stem cells (MSCs). Further study is warranted to determine if subsets of patients 
respond differently to this treatment such as older/younger diabetic/nondiabetic, male/female, smoker/nonsmoker subject pairs.

\section{References}

1. Philpin B, Ganglof P, Tran N (2009) Rehabilitation of irradiated head and neck tissues by autologous fat transplantation. Plast Reconstr Surg 123(4): 1187-1197.

2. Karmali R, Hanson S, Ngyuyen A, et al. (2018) Outcomes following autologous fat transfer for oncological head and neck reconstruction. Plast Reconstr Surg 142(3): 771-780.

3. Rigotti G, Marchi A, Gailie M (2007) Clinical treatment of radiotherapy tissue damage by lipoaspirate transplant: a healing process mediated by adipose derived adult stem cells. Plast Reconstr Surg 119(5): 14091422.

4. Cervelli V, Gentile P, Grimaldi M (2009) regenerative surgery: use of fat grafts combined with platelet rich plasma for lower extremity ulcers. Aesthetic Plast Surg 33(3): 340-345.

5. Bura A, Planet-Bernard V, Bourin P (2014) The use of autologous cultured cells adipose stroma/stem cells to treat patients with non vascularizeable critical limb ischemia. Cytotherapy 16(2): 245-257.

6. Gentile P, Orlandi A, Scioli M (2012) Concise review: adipose derived stromal vascular fraction cells and platelet rich plasma: basic and clinical implications for tissue engineering therapies in regenerative surgery. Stem Cells Transl Med 1(3): 230-236.

7. Blanton MW, Hada I, Johnstone BH (2009) Adipose stromal cells and platelet rich plasma therapies synergistically increase revascularization during wound healing. Plast Reconstr Surg 123(2): 56S-64S.
8. Cervelli V, Scioli M, Gentile P, Doldo E, Bonanno E, et al. (2012) Platelet rich plasma greatly potentiates insulin induced adipogenic differentiation of human adipose derived stem cells through a serine/threonine Akt dependent mechanism and promotes clinical graft maintenance. Stem Cells Transl Med 1(3): 206-220.

9. D Amico R, Rubin P, Neumeister M (2013) A Report of the ASPS task force on regenerative medicine: opportunities for plastic surgery. 131(2): 393-399.

10. Bowen R (2016) Stromal vascular fraction from lipoaspirate infranatant: comparison between suction assisted liposuction and nutational infrasonic liposucton. Aesthetic Plast Surg 40(3): 367-371.

11. Yoshimura K, Shiguera T, Matsumoto D (2006) Characterization of freshly isolated and cultured cells derived from fluid and fatty portions of lipoaspirate. J Cell Physio 208: 64-76.

12. Nie C, Yang D, Xu J (2016) Locally administered adipose derived stem cells accelerate wound healing through differentiation and vasculogenesis. Cell Transplant 20(2): 205-216.

13. Matsumoto D, Sato K, Gonda (2006) Cell assisted lipotranfer: supportive use of human adipose derived stem cells for soft tissue augmentation with lipoinjection. Tissue Eng 12(12): 3375-3382.

14. Pallua N, Pulsfort A, Suschek N (2009) Content of growth factors bFGF, IGF-1, VEGF, and PDGG-BB in freshly harvested lipoaspirate after centrifugation and incubation. Plast Reconstr Surg 123: 826-833.

15. Rehman J, TraktuevD, Li J (2004) Secretion of angiogenic and antiapoptotic factors by human adipose stromal cells. Circulation 109: 1292-1298.

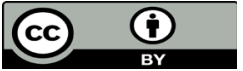

This work is licensed under Creative Commons Attribution 4.0 License

To Submit Your Article Click Here: Submit Article

DOI: $10.32474 /$ SCSOAJ.2019.02.000148

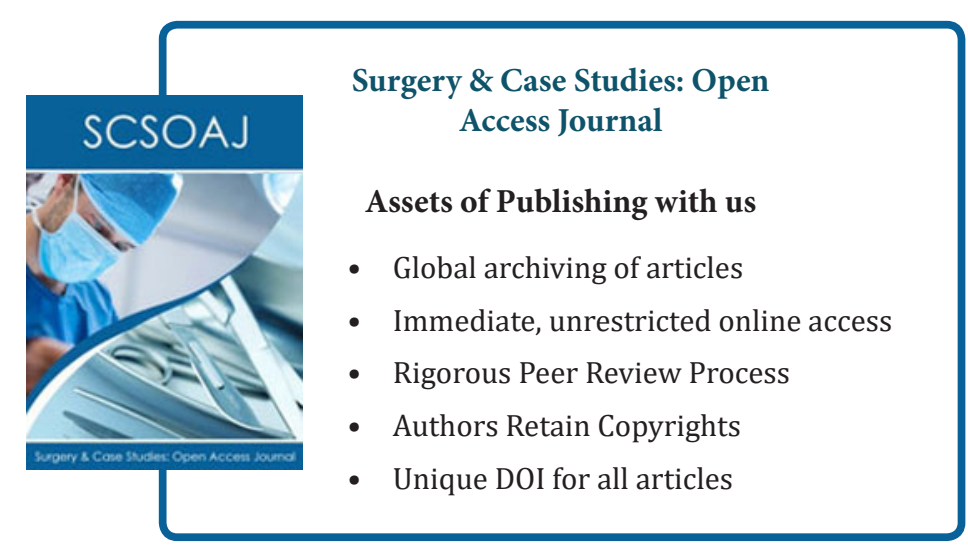

